\title{
Nefropatia associata al SARS-CoV-2: cosa sappiamo finora
}

\author{
Aris Tsalouchos ${ }^{1}$, Maurizio Salvadori ${ }^{2}$ \\ ${ }^{1}$ Azienda UsI Toscana Centro, S.O.S. Nefrologia e Dialisi, Ospedale SS. Cosma e Damiano, Pescia, Pistoia - Italia \\ ${ }^{2}$ Professore di Nefrologia, già Direttore Nefrologia e Trapianto, Azienda Ospedaliera Careggi, Firenze - Italia
}

\begin{abstract}
SARS-CoV-2 associated nephropathy: what we know so far
Acute kidney injury (AKI) is a frequent complication of severe acute respiratory syndrome coronavirus 2 (SARSCoV-2) attributable to i) hypotension and decreased kidney perfusion secondary to hemodynamic or hemostatic factors, ii) drug-induced nephrotoxicity, iii) cytokine storm syndrome related to sepsis. However: i) early new-onset proteinuria and hematuria in many patients, ii) the identification of SARS-CoV-2 viral load in precisely defined kidney compartments, iii) ultrastructural evidence of direct viral infection of the kidneys, and most importantly, iv) morphological alterations associated to cytopathic action induced by the virus support the existence of SARSCoV-2 associated nephropathy. In addition, collapsing glomerulopathy reported in African American patients with underlying APOL1 kidney risk alleles and SARS-CoV-2 infection is the evidence of a distinct form of SARS-CoV-2 associated nephropathy, the APOL1-SARS-CoV2-associated nephropathy.
\end{abstract}

Keywords: APOL1-SARS-CoV2-associated nephropathy, SARS-CoV-2, SARS-CoV-2-associated nephropathy

\section{Introduzione}

La prima segnalazione ufficiale di casi di polmonite di origine sconosciuta, successivamente attribuibili al Severe Acute Respiratory Syndrome Coronavirus 2 (SARS-CoV-2) è avvenuta il 31 dicembre 2019 nella città di Wuhan, capoluogo della provincia cinese dell'Hubei (1). In data 11 marzo, I'Organizzazione Mondiale della Sanità (OMS) ha dichiarato lo stato di pandemia, dopo che, nelle precedenti due settimane, il numero di casi di SARS-CoV-2 al di fuori della Cina è aumentato di 13 volte e il numero di paesi colpiti è triplicato (più di 118.000 casi in 114 paesi e di 4291 persone decedute) (2).

Al momento dell'invio del'articolo (11 giugno), con la pandemia in corso, i casi confermati nel mondo sono 7.221.717 e i morti 411.818 con 34.167 di questi in Italia (3).

I coronavirus sono una vasta famiglia di virus, ma solo sei (HCoV-229E, HCoV-NL63, HCoV-OC43, HCoV-KU1, MERS-CoV, SARS-CoV) erano precedentemente noti per la capacità di

Received: June 12, 2020

Accepted: June 16, 2020

Published online: July 08, 2020

Indirizzo per la corrispondenza:

Aris Tsalouchos

Azienda Usl Toscana Centro

S.O.S. Nefrologia e Dialisi

Ospedale SS. Cosma e Damiano

Via Cesare Battisti 2

51017 Pescia, Pistoia - Italia

aris.tsalouchos@uslcentro.toscana.it infettare l'uomo; quindi, il SARS-CoV-2 è il settimo (4). II SARSCoV-2, insieme al SARS-CoV e al MERS-CoV, è un ceppo virale della specie SARS-related coronavirus, membro del genere Betacoronavirus, sottogenere Serbecovirus (5).

Andersen et al. (6) hanno proposto due possibili scenari riguardanti le origini di selezione naturale del SARS-CoV-2, poste temporalmente prima del salto di specie dall'animale all'uomo e dopo, non escludendo, però, una terza ipotesi: la selezione naturale del SARS-CoV-2 in colture cellulari e/o in modelli animali durante i passaggi in laboratorio (6).

La trasmissione interumana del SARS-CoV-2 avviene principalmente attraverso il contatto con le goccioline del respiro (droplets) di persone infette (7). Tuttavia, l'isolamento del virus nelle feci e in liquidi biologici (8), perfino nel liquido peritoneale di pazienti infetti (9), non escluderebbe rari casi di contagio attraverso materiale biologico diverso dalle droplets.

La sintomatologia, dopo un periodo di incubazione che varia fra i 2 e i 14 giorni $(10,11)$, può essere estremamente variabile, da lieve a grave. Nell' $80 \%$ dei casi, i soggetti infetti sono asintomatici, pauci-sintomatici e con sintomatologia lieve caratterizzata da febbre, mal di gola, tosse secca, congiuntivite, stanchezza, diarrea, anosmia/iposmia e, in alcuni casi ageusia (12). II 15-20\% dei pazienti, a distanza circa di 1 settimana dalla comparsa dei primi sintomi, progredisce verso una malattia più grave caratterizzata inizialmente da dispnea ingravescente, manifestazione clinica di polmonite interstiziale e, infine, SARS, con necessità di ventilazione meccanica $(13,14)$.

L'impegno delle basse vie respiratorie non è l'unica complicanza d'organo severa in corso di infezione da SARS-CoV-2. 
Molti organi e apparati vengono coinvolti, determinando una sindrome da disfunzione mutliorgano (MODS), in buona parte dovuta alla sindrome da rilascio di citochine (CRS) $(14,15)$. Tuttavia, serie di autopsie dimostrano che il SARS-CoV-2 ha un organotropismo che va oltre il tratto respiratorio, includendo reni, cuore, fegato e cervello (16), anche se non è chiaro se gli effetti citopatici virali diretti in questi siti contribuiscano alle complicazioni osservate.

In questo articolo riportiamo i dati epidemiologici e clinici, patofisiologici e, soprattutto, istopatologici che supportano un diretto danno renale da SARS-CoV-2, per poter parlare di nefropatia associata al SARS-CoV-2 o SARS-CoV-2 nephropathy.

\section{Epidemiologia e clinica del coinvolgimento renale}

Dati iniziali hanno suggerito una bassa incidenza (3-9\%) di danno renale acuto (AKI) in pazienti affetti da SARS-CoV-2 $(10,13,17,18)$. Tuttavia, dati più recenti riportano l'incidenza di AKI nel 27-37\% dei pazienti ospedalizzati $(19,20)$. In un'ampia casistica di pazienti (5449), ricoverati con SARS-CoV-2 nella città metropolitana di New York, l'AKI si è sviluppato nel $37 \%$ dei casi (20). Secondo i criteri diagnostici KDIGO (Kindey Disease: Improving Global Outcomes) (21) per l'AKI, il 47\% dei pazienti ha sviluppato AKI di stadio I, il $22 \%$ AKI di stadio II e il $31 \%$ AKI di stadio III (20).

II $15 \%$ dei pazienti con AKI ha richiesto un trattamento emodialitico e, di questi, il $97 \%$ era già in ventilazione meccanica. L'AKI si è sviluppato nel $90 \%$ dei pazienti in ventilazione meccanica, mentre, nei pazienti non critici, era meno frequente (22\%) (20). Inoltre, l'associazione temporale tra I'inizio della ventilazione meccanica e lo sviluppo dell'AKI era molto stretta (il 52\% dopo $24 \mathrm{~h}$ dall'intubazione) (20).

Lo sviluppo di AKI nei pazienti con SARS-CoV-2 è un fattore di rischio indipendente di mortalità intraospedaliera, come dimostrato in due casistiche cinesi $(18,19)$. Nell'ampia casistica di New York, la mortalità dei pazienti con AKI era del $35 \%$, il $26 \%$ dei pazienti è stato dimesso, mentre il 39\% dei pazienti con AKI risultava ancora ricoverato al momento della pubblicazione dello studio (20). L'analisi multivariata della casistica di New York ha evidenziato i seguenti fattori come predittivi indipendenti per lo sviluppo di AKI: età avanzata, discendenza africana, diabete mellito, ipertensione, malattie cardiovascolari, ventilazione meccanica e uso di farmaci vasopressori (20). In questa analisi, gli inibitori del sistema reninaangiotensina-aldosterone (RAASi) non risultavano predittivi di AKI (20), confermando il consiglio di molte società scientifiche e di singoli esperti che il loro utilizzo non deve essere interrotto per motivi di profilassi (22).

L'esatto meccanismo del coinvolgimento e del danno renale in corso di SARS-CoV-2 non è chiaro. Meccanismi postulati includono $(23,24)$ : AKI pre-renale in corso di MODS (soprattutto in pazienti ricoverati in rianimazione e con precedente ridotta riserva funzionale renale), AKI iatrogena da uso di farmaci nefrotossici, la sindrome da rilascio di citochine (CRS), che potrebbe determinare una nefrite tubulo-interstiziale, e, infine, un danno cellulare diretto del virus a livello tubulare e podicitario.

A favore dell'ipotesi del danno virale diretto e, quindi, della SARS-CoV-2 nephropathy ci sono vari elementi clinici, prima di quelli istopatologici. Cheng et al. (18), in una casistica di 701 pazienti, hanno riportato che, all'ingresso in ospedale, il $44 \%$ dei pazienti presentava proteinuria e il $27 \%$ microematuria. Lo stesso studio ha riportato una bassa incidenza di AKI intraospedaliero (5\%) di questi pazienti, facendo, così, supporre, in assenza di dati istologici, che l'alta percentuale delle anomalie urinarie riscontrate all'ingresso fosse determinata da un danno glomerulare diretto dal virus (18). Hong et al. (25), nel loro intento di identificare e valutare l'incidenza del danno renale precoce in 12 pazienti con SARS-CoV-2, hanno analizzato le urine dei pazienti riscontrando albuminuria nel $42 \%$ dei casi, senza significative alterazioni nei valori di azotemia e creatinina plasmatica associate. II dato, però, più significativo del danno glomerulare era il riscontro di proteinuria non selettiva caratterizzata dalla presenza di lgG e transferrina nelle urine, mentre, a indicare il danno tubulare, c'era il riscontro nelle urine di a1-microglobulina (25). A differenza degli studi precedenti, l'alta percentuale di microematuria (46\%) e di proteinuria (42\%) riscontrata nei pazienti con AKI nell'ampia casistica di New York non può essere presa in considerazione per un eventuale danno glomerulare, in quanto gli autori non erano in grado di dire se i pazienti erano portatori di catetere vescicale al momento della raccolta dell'esame urine (20). Inoltre, oltre alle anomalie urinarie riscontrate in molti pazienti nella fase precoce della malattia, il riscontro di particelle virali (26) e, ancora, di RNA virale (27) nelle urine di alcuni pazienti è un indicatore indiretto di SARS-CoV-2 nephropathy.

\section{Fisiopatologia dell’organotropismo renale}

II SARS-CoV-2, cosi come il SARS-CoV, entra nelle cellule del nostro organismo attraverso il legame della sua proteina spike e del recettore cellulare angiotensin-converting enzyme 2 (ACE2) (28). II recettore ACE2 non è espresso soltanto sulla membrana cellulare dei pneumociti di tipo 2, ma è quasi ubiquitario, essendo stato isolato sulla mucosa orale e nasale, nel nasofaringe, nell'intero tratto gastrointestinale e in linfonodi, timo, midollo osseo, milza, fegato, reni, cervello, vasi e cuore $(29,30)$. In particolare, l'espressione dell'ACE2 a livello renale risulta essere maggiore che a livello polmonare $(31,32)$. L'ACE2 è espresso principalmente in sede apicale delle cellule tubulari prossimali e, in minor misura, sulla superficie dei podociti (32).

Si ipotizza che la maggiore tendenza del SARS-CoV-2 a causare una grave infezione a livello bronchiolo-alveolare, rispetto ad altri organi e apparati, sia dovuta al fatto che l'infettività cellulare non dipende soltanto dall'espressione cellulare 
dell'ACE2. L'ingresso del SARS-CoV-2 nella cellula attraverso il recettore ACE2 è "aiutato" da alcune proteasi situate sulla superficie cellulare, con probabile maggiore espressione a livello alveolare, mentre altre proteasi facilitano la diffusione del virus una volta che questo è penetrato all'interno della cellula. In particolare, la transmembrane protease serine 2 (TMPRSS2), membro della sottofamiglia Hepsin-TMPRSS, è un enzima proteolitico transmembrana, che, anche se stechiometricamente separato, strutturalmente e funzionalmente fa parte del recettore ACE2. La TMPRSS2 si lega con la sub-unita S1 della proteina spike virale e la distacca dalla sub-unità S2. Tale attività enzimatica aumenta di quasi 100 volte l'ingresso del virus nella cellula attraverso il recettore ACE2, in quanto, a distacco avvenuto, l'unità S2 virale si fonde con la cellula e, attraverso tale unità, avviene il trasferimento del contenuto virale nel citoplasma (33-35).

La TMPRSS2 nel rene è maggiormente espressa a livello delle cellule tubulari distali che nelle cellule tubulari prossimali (36-38). Resta, comunque, da determinare se altre TMPRSS, come le TMPRSS4, 5 e 9, situate a livello delle cellule tubulari prossimali possano mediare l'ingresso cellulare del SARS-CoV-2.

Le cellule glomerulari endoteliali, che rappresentano la prima linea di difesa, possono essere direttamente infettate dal SARS-CoV-2, come, peraltro, è stato dimostrato con l'aiuto della microscopia elettronica in un paziente trapiantato di rene (39). Successivamente, il virus potrebbe invadere i podociti; particelle virali sono state identificate nel citoplasma podocitario $(40,41)$ e, infine, passando nello spazio di Bowman, arrivano a infettare le cellule tubulari prossimali e distali, come dimostrato dalla presenza di particelle virali anche a livello citoplasmatico di cellule tubulari prossimali $(41,42)$.

La diversa incidenza di AKI osservata tra pazienti asiatici e pazienti di origine occidentale potrebbe essere spiegata da una maggiore espressione di ACE2 e proteasi della famiglia TMPRSS a livello renale di soggetti con origine occidentale, come è stato dimostrato in un recente studio di analisi trascrittomica di 15 campioni di rene umano sano (43).

\section{Istopatologia renale e SARS-CoV-2 nephropathy}

I primi dati di istopatologia renale provengono da 26 autopsie di pazienti infetti da SARS-CoV-2 (41). Alla microscopia ottica, tutti i campioni analizzati presentavano necrosi tubulare acuta (ATN) prossimale di vario grado, caratterizzata da perdita dell'orletto a spazzola e da degenerazione vacuolare. Occasionali granuli di emosiderina nelle cellule tubulari erano osservati in 4 pazienti, con presenza di microematuria e quadro clinico di rabdomiolisi. L'interstizio era indenne da infiltrazione cellulare. I glomeruli presentavano varie alterazioni caratterizzate da segni di nefropatia diabetica in 2 pazienti diabetici e dalla presenza di glomeruli ischemici in 11 pazienti ipertesi. I capillari glomerulari e peritubulari si mostravano ostruiti da aggregati di eritrociti in assenza di piastrine o trombi di fibrina nel loro lume. Alla microscopia elettronica, erano presenti particelle virali nel citoplasma delle cellule tubulari prossimali e dei podociti, mentre erano meno evidenti a livello delle cellule tubulari distali. Sempre con la microscopia elettronica, nei punti dove erano presenti particelle virali citoplasmatiche, era possibile osservare una segmentaria fusione dei pedicelli podocitari e una vacuolizzazione e un distacco occasionali dei podociti dalla membrana glomerulare. Le particelle virali a livello tubulare erano identificate anche con l'immunofluorescenza indiretta utilizzando anticorpi diretti contro nucleoproteine comuni tra i betacoronavirus, mentre, con l'immunoistochimica, l'espressione di ACE2 si mostrava upregolata, in particolar modo nelle sedi con severa ATN. Nel loro insieme, queste osservazioni dimostrano che, in parte, il quadro di ATN e la presenza di proteinuria di nuovo riscontro possono essere causati da un danno virale diretto.

Diao et al. (44), in modo analogo, analizzando post-mortem 6 campioni di tessuto renale, hanno trovato segni di ATN di vario grado in tutti i campioni. A differenza, però, con le osservazioni di Su et al. (41), a livello tubulo-interstiziale erano presenti infiltrati cellulari in 5 dei 6 campioni analizzati, con 2 di questi di grado severo. All'immunoistochimica, nella grande maggioranza queste cellule erano dei macrofagi $\mathrm{CD}^{2} 8^{+}$, mentre linfociti $\mathrm{CD} 8^{+}$erano presenti in grado moderato. Invece, la presenza di linfociti $\mathrm{CD}^{+}$e di natural killer $\mathrm{CD}^{2} 6^{+}$era scarsa. L'effetto citopatico diretto del SARS-CoV-2 è stato osservato in 3 campioni in presenza di sincizi cellulari. Alla microscopia ottica, i glomeruli non presentavano significative alterazioni, a parte segni di glomerulosclerosi focale segmentaria con immunofluorescenza negativa per depositi di immunoglobuline in 3 pazienti ipertesi. L'indagine di immunoistochimica ha rilevato, inoltre, l'espressione di antigeni del nucleocapside virale limitata a livello citoplasmatico delle cellule tubulari, in tutti i campioni analizzati. Alcune delle cellule tubulari, positive per l'espressione di antigeni del nucleocapside virale, avevano anche perso il loro contatto con l'epitelio tubulare. Infine, con l'immunoistochimica è stato possibile evidenziare la presenza di un altro importante "attore" corresponsabile del danno renale, in quanto l'attivazione della cascata complementare a livello locale è stata rilevata da una forte positività per il membrane-attack complex (MAC; C5b-9) a livello tubulare di tutti i campioni, mentre una blanda positività è stata riscontrata a livello glomerulare in solo 2 casi. Un limite del lavoro di Diao et al. (44) è stato l'assenza di indagine ultrastrutturale. L'importanza della cascata complementare nella patogenesi sistemica del SARS-CoV-2 viene riportata in una review da Noris et al. (45), mentre Jhaveri et al. (46) hanno descritto il primo caso di sindrome uremico emolitica complemento mediata con diffusa necrosi corticale. II giorno dopo l'esecuzione della biopsia renale è stata effettuata una singola dose di eculizumab (900 mg) e il paziente è deceduto 2 giorni dopo in corso di insufficienza multiorgano (46). 
Oltre al danno infiammatorio sistemico a carico dei vasi, determinato dal rilascio di citochine e dall'attivazione della cascata complementare, le cellule endoteliali rappresentano un target preferenziale del SARS-CoV-2. Varga et al. (39) hanno per primi dimostrato, con un'indagine ultrastrutturale, la presenza di inclusioni virali anche in cellule dell'endotelio glomerulare, oltre ai casi già descritti a carico delle cellule tubulari e nei podociti.

In letteratura, per il momento, sono stati descritti 3 case report $(40,47,48)$ di pazienti di origine africana $(2$ maschi e 1 femmina) con AKI, proteinuria nefrosica e quadro istopatologico di collapsing glomerulopathy. Due dei pazienti erano portatori dell'allele G1 del gene APOL1 $(47,48)$, cosa che non si può escludere anche per il terzo paziente, il quale non ha effettuato l'indagine genetica (40). Notoriamente, pazienti portatori degli alleli G1 e G2 del gene APOL1, nella loro grande maggioranza di discendenza africana subsahariana, sono suscettibili di CKD e glomerulopatie, come la glomerulosclerosi focale segmentaria (FSGS) e l'HIV-associated nephropathy, in modo tale che, negli ultimi anni, è diffusamente utilizzato il termine di APOL1-associated nephropathy (49). La descrizione di casi di collapsing glomerulopathy in pazienti con SARS-CoV-2 di origine africana e portatori del allele G1 del gene APOL1 potrebbe, quindi, spiegare perché la discendenza africana rappresenti uno dei fattori predittivi indipendenti per lo sviluppo di AKI.

Infine, oltre alle caratteristiche istopatologiche renali appena descritte, la dimostrazione più convincente del tropismo renale del SARS-CoV-2 viene da Puelles et al. (16), che hanno per primi quantificato direttamente la carica virale in campioni tissutali di vari organi provenienti da pazienti deceduti. Le cariche virali più alte erano provenienti da campioni del tratto respiratorio, mentre valori più bassi erano riscontrati a rene, fegato, cuore e cervello (16). In particolare, 3 dei 6 campioni di tessuto renale analizzati hanno mostrato la presenza di carica virale in tutti i compartimenti anatomici, ma il target preferenziale del virus era rappresentato dalle cellule glomerulari.

\section{Conclusioni}

Il danno renale in corso di infezione da SARS-CoV-2 è multifattoriale. Lo stato emodinamico precario e l'età avanzata di molti pazienti con AKI, insieme allo stato infiammatorio sistemico come risposta organica all'infezione virale, rappresentano le cause principali dell'interessamento renale. Dall'altra parte, però, dobbiamo sottolineare il preferenziale organotropismo che mostra il virus nei confronti del rene. II riscontro di RNA virale nelle urine e, soprattutto, in campioni di tessuto renale provenienti da pazienti deceduti è la prova certa del tropismo renale, ma non dimostra il suo effetto citopatico diretto per poter parlare di SARS-CoV-2 nephropathy. Segni indiretti di danno citopatico renale rappresentano le anomalie urinarie, proteinuria e microematuria, nelle fasi precoci della malattia e il riscontro di particelle virali a livello citoplasmatico di cellule tubulari prossimali, cellule endoteliali glomerulari e podociti. Segni diretti di danno citopatico e, quindi, di SARS-CoV-2 nephropathy presentano, invece, le alterazioni morfologiche riscontrate in cellule renali con inclusioni virali, e queste sono la degenerazione vacuolare, la presenza di sincizi cellulari, la fusione dei pedicelli podocitari e, infine, la presenza di cellule tubulari e di podociti distaccati dalle loro membrane di sostegno. La presenza di quadri istopatologici di collapsing glomerulopathy in pazienti con discendenza africana subsahariana portatori del allele G1 del gene APOL1 ci permette, inoltre, di utilizzare il termine di APOL1-SARS-CoV-2-associated nephropathy.

\section{Disclosures}

Conflict of interest: The authors declare no conflict of interest. Financial support: This research received no specific grant from any funding agency in the public, commercial, or not-for-profit sectors. Authors contribution: All authors contributed equally to this manuscript.

\section{Bibliografia}

1. Pneumonia of unknown cause - China. Disease outbreak news, World Health Organization, 5 January 2020. Available at: https://www.who.int/csr/don/05-january-2020-pneumoniaof-unkown-cause-china/en/. Accessed April 27, 2020.

2. L'Organizzazione Mondiale della Sanità dichiara il coronavirus pandemia. http://www.salute.gov.it/portale/nuovocoronavirus/dettaglioNotizieNuovoCoronavirus.jsp?lingua=italiano\& menu=notizie $\& p=$ dalministero\&id=4209. Accessed April 27, 2020.

3. Covid-19 - Situazione nel mondo. http://www.salute.gov.it/ portale/nuovocoronavirus/dettaglioContenutiNuovoCoronavirus. jsp?lingua=italiano\&id $=5338 \&$ area $=$ nuovoCoronavirus $\&$ menu $=$ vuoto. Accessed June 11, 2020.

4. Corman VM, Muth D, Niemeyer D, et al. Hosts and Sources of Endemic Human Coronaviruses. Adv Virus Res. 2018; 100:163-88

5. Sun P, Lu X, Xu C, et al. Understanding of COVID-19 based on current evidence, published online ahead of print, 2020 Feb 25. J Med Virol. 2020;10.1002/jmv.25722.

6. Andersen KG, Rambaut A, Lipkin $\mathrm{WI}$, et al. The proximal origin of SARS-CoV-2. Nat Med. 2020;26(4):450-2.

7. Miller R, Englund K. Transmission and risk factors of COVID-19, published online ahead of print, 2020 May 14. Cleve Clin J Med. 2020;10.3949/ccjm.87a.ccc029.

8. Wang W, Xu Y, Gao R, et al. Detection of SARS-CoV-2 in Different Types of Clinical Specimens, published online ahead of print, 2020 Mar 11. JAMA. 2020;323(18):1843-4.

9. Vischini G, D'Alonzo S, Grandaliano G, et al. SARS-CoV-2 in the peritoneal waste in a patient treated with peritoneal dialysis, published online ahead of print, 2020 May 12. Kidney Int. 2020;S0085-2538(20):30531-7.

10. Guan WJ, Ni ZY, Hu Y, et al. Clinical Characteristics of Coronavirus Disease 2019 in China. N Engl J Med. 2020;382(18):1708-20.

11. Lauer SA, Grantz $\mathrm{KH}, \mathrm{Bi} \mathrm{Q}$, et al. The Incubation Period of Coronavirus Disease 2019 (COVID-19) From Publicly Reported Confirmed Cases: Estimation and Application. Ann Intern Med. 2020;172(9):577-82.

12. Wu Z, McGoogan JM. Characteristics of and Important Lessons From the Coronavirus Disease 2019 (COVID-19) Outbreak 
in China: Summary of a Report of 72314 Cases From the Chinese Center for Disease Control and Prevention, published online ahead of print, 2020 Feb 24. JAMA. 2020;10.1001/ jama.2020.2648.

13. Wang D, Hu B, Hu C, et al. Clinical Characteristics of 138 Hospitalized Patients With 2019 Novel Coronavirus-Infected Pneumonia in Wuhan, China, published online ahead of print, 2020 Feb 7. JAMA. 2020;323(11):1061-9.

14. Huang $C$, Wang $Y$, Li X, et al. Clinical features of patients infected with 2019 novel coronavirus in Wuhan, China, published correction appears in Lancet. 2020 Jan 30. Lancet. 2020;395(10223):497-506

15. Moore JB, June $\mathrm{CH}$. Cytokine release syndrome in severe COVID-19. Science. 2020;368(6490):473-4.

16. Puelles VG, Lütgehetmann M, Lindenmeyer MT, et al. Multiorgan and Renal Tropism of SARS-CoV-2, published online ahead of print, 2020 May 13. N Engl J Med. 2020;NEJMc2011400. doi:10.1056/NEJMc2011400.

17. Chen N, Zhou M, Dong X, et al. Epidemiological and clinical characteristics of 99 cases of 2019 novel coronavirus pneumonia in Wuhan, China: a descriptive study. Lancet. 2020;395(10223):507-13.

18. Cheng $\mathrm{Y}$, Luo $\mathrm{R}$, Wang $\mathrm{K}$, et al. Kidney disease is associated with in-hospital death of patients with COVID-19. Kidney Int. 2020;97(5):829-38.

19. Li Z, Wu M, Guo J, et al. Caution on kidney dysfunctions of 2019-nCoV patients. medRxiv 2020.02.08.20021212. Accessed March 2, 2020.

20. Hirsch JS, Ng JH, Ross DW, et al. Acute kidney injury in patients hospitalized with COVID-19, published online ahead of print, 2020 May 16. Kidney Int. 2020;S0085-2538(20)30532-9.

21. Khwaja A. KDIGO clinical practice guidelines for acute kidney injury. Nephron Clin Pract. 2012;120(4):c179-84.

22. Vaduganathan $M$, Vardeny $O$, Michel $T$, et al. Renin-AngiotensinAldosterone System Inhibitors in Patients with Covid-19. N Engl J Med. 2020;382(17):1653-9.

23. Naicker S, Yang CW, Hwang SJ, et al. The Novel Coronavirus 2019 epidemic and kidneys. Kidney Int. 2020;97(5):824-8.

24. Batlle D, Soler MJ, Sparks MA, et al. Acute Kidney Injury in COVID-19: Emerging Evidence of a Distinct Pathophysiology, published online ahead of print, 2020 May 4. J Am Soc Nephrol. 2020;ASN.2020040419.

25. Hong Xw, Chi Zp, Liu Gy, et al. Analysis of early renal injury in COVID-19 and diagnostic value of multi-index combined detection. MedRxiv. 2020. doi: 10.1101/2020.03.07.20032599. Accessed May 25, 2020.

26. Zhou P, Yang XL, Wang XG, et al. A pneumonia outbreak associated with a new coronavirus of probable bat origin. Nature. 2020;579:270-3.

27. Gross O, Moerer O, Weber M, et al. COVID-19-associated nephritis: early warning for disease severity and complications? Lancet. 2020;395(10236):e87-8.

28. Wan Y, Shang J, Graham R, et al. Receptor Recognition by the Novel Coronavirus from Wuhan: an Analysis Based on Decade-Long Structural Studies of SARS Coronavirus. J Virol. 2020;94(7):e00127-20.

29. Hamming I, Timens W, Bulthuis ML, et al. Tissue distribution of ACE2 protein, the functional receptor for SARS coronavirus. A first step in understanding SARS pathogenesis. J Pathol. 2004;203(2):631-7.

30. Guo L, Yu K, Li D, et al. Potential Pathogenesis of Multiple Organ Injury in COVID-19. Preprints 2020. doi: 10.20944/ preprints202003.0308.v1.

31. Serfozo P, Wysocki J, Gulua G, et al. Ang II (Angiotensin II) Conversion to Angiotensin-(1-7) in the Circulation Is POP
(Prolyloligopeptidase)-Dependent and ACE2 (AngiotensinConverting Enzyme 2)-Independent. Hypertension. 2020; 75(1):173-82.

32. Ye M, Wysocki J, William J, et al. Glomerular localization and expression of Angiotensin-converting enzyme 2 and Angiotensin-converting enzyme: implications for albuminuria in diabetes. J Am Soc Nephrol. 2006;17(11):3067-75.

33. Hoffmann M, Kleine-Weber $\mathrm{H}$, Schroeder $\mathrm{S}$, et al. SARS-CoV-2 Cell Entry Depends on ACE2 and TMPRSS2 and Is Blocked by a Clinically Proven Protease Inhibitor. Cell. 2020;181(2):271-80.e8.

34. Matsuyama S, Ujike M, Morikawa S, et al. Protease-mediated enhancement of severe acute respiratory syndrome coronavirus infection. Proc Natl Acad Sci U S A. 2005;102(35):12543-7.

35. Shulla A, Heald-Sargent T, Subramanya G, et al. A transmembrane serine protease is linked to the severe acute respiratory syndrome coronavirus receptor and activates virus entry. J Virol. 2011;85(2):873-82.

36. Wu H, Uchimura K, Donnelly EL, et al. Comparative Analysis and Refinement of Human PSC-Derived Kidney Organoid Differentiation with Single-Cell Transcriptomics. Cell Stem Cell. 2018;23(6):869-81.e8.

37. Wilson PC, Wu H, Kirita $\mathrm{Y}$, et al. The single-cell transcriptomic landscape of early human diabetic nephropathy. Proc Natl Acad Sci U S A. 2019;116(39):19619-25.

38. Walls AC, Park YJ, Tortorici MA, et al. Structure, Function, and Antigenicity of the SARS-CoV-2 Spike Glycoprotein. Cell. 2020;181(2):281-92.e6.

39. Varga Z, Flammer AJ, Steiger $P$, et al. Endothelial cell infection and endotheliitis in COVID-19. Lancet. 2020;395(10234):1417-8.

40. Kissling S, Rotman S, Gerber C, et al. Collapsing glomerulopathy in a COVID-19 patient, published online ahead of print, 2020 Apr 15. Kidney Int. 2020;S0085-2538(20)30395-1.

41. Su H, Yang M, Wan C, et al. Renal histopathological analysis of 26 postmortem findings of patients with COVID-19 in China. Kidney Int 2020; 98:219-27.

42. Farkash EA, Wilson AM, Jentzen JM. Ultrastructural Evidence for Direct Renal Infection with SARS-CoV-2, published online ahead of print, 2020 May 5. J Am Soc Nephrol. 2020;ASN.2020040432.

43. Pan $X W, X u D$, Zhang $H$, et al. Identification of a potential mechanism of acute kidney injury during the COVID-19 outbreak: a study based on single-cell transcriptome analysis, published online ahead of print, 2020 Mar 31. Intensive Care Med. 2020;1-3.

44. Diao B, Wang C, Wang R, et al. Human Kidney is a Target for Novel Severe Acute Respiratory Syndrome Coronavirus 2 (SARSCoV-2) Infection. medRxiv 2020.03.04.20031120. Accessed April 25, 2020.

45. Noris M, Benigni A, Remuzzi G. The case of Complement activation in COVID-19 multiorgan impact, published online ahead of print, 2020 May 24. Kidney Int. 2020;S0085-2538 (20):30556-1.

46. Jhaveri KD, Meir LR, Flores Chang BS, et al. Thrombotic microangiopathy in a patient with COVID-19. Kidney International (2020), doi: https://doi.org/10.1016/j.kint.2020.05.025.

47. Larsen CP, Bourne TD, Wilson JD, et al. Collapsing Glomerulopathy in a Patient With Coronavirus Disease 2019 (COVID-19), published online ahead of print, 2020 Apr 9. Kidney Int Rep. 2020;5(6):935-9.

48. Peleg Y, Kudose S, D'Agati V, et al. Acute Kidney Injury Due to Collapsing Glomerulopathy Following COVID-19 Infection, published online ahead of print, 2020 Apr 28. Kidney Int Rep. 2020;5(6):940-5.

49. Freedman BI, Limou S, Ma L, Kopp JB. APOL1-Associated Nephropathy: A Key Contributor to Racial Disparities in CKD. Am J Kidney Dis. 2018;72(5 Suppl. 1):S8-16. 